\title{
Finite direct sums of cyclic embeddings
}

\author{
Justyna Kosakowska and Markus Schmidmeier \\ Dedicated to Professor José Antonio de la Peña
}

\begin{abstract}
In this paper we generalize Kaplansky's combinatorial characterization of the isomorphism types of embeddings of a cyclic subgroup in a finite abelian group given in his 1951 book "Infinite Abelian Groups". For this we introduce partial maps on Littlewood-Richardson tableaux and show that they characterize the isomorphism types of finite direct sums of such cyclic embeddings.
\end{abstract}

MSC 2010: 05E10, 20K27, 47A15

Key words: subgroup embedding; invariant subspace; Littlewood-Richardson tableau; partial map

\section{Introduction}

In [1] Birkhoff stated the problem of classification of subgroups of finite abelian groups. Connected with this problem are results of Kaplansky in [4] that present a combinatorial characterization of the isomorphism types of embeddings of a cyclic subgroup in a finite abelian group. This characterization uses sequences of positive integers, see Section 2.1 for details. In this paper we use partial maps on Littlewood-Richardson tableaux (LR-tableaux for short) to generalize this result to finite direct sums of such embeddings. We extend results given in [8, Section 2] and present some applications.

Instead of finite abelian groups we work with finitely generated modules over a discrete valuation ring $\Lambda$. One of the presented result is Theorem 3.1 saying that a direct sum of cyclic embeddings is uniquely determined by the associated LR-tableau with a partial map satisfying the empty box property, see Section 2.2 for definitions. LR-tableaux were successfully used in [6, 7, 8, 9] to describe algebraic and geometric properties of short exact sequences of $\Lambda$-modules. Results obtained there show that relationships between short extact sequences of $\Lambda$-modules and LR-tableaux are deep and interesting. This motivates us to collect properties of LR-tableaux of direct sums of cyclic embeddings. 
The paper is organized as follows.

In Section 2 we present notation, definitions and basic facts concerning cyclic embeddings. In particular, we recall results of Kaplansky and rewrite them in the language of Littlewood-Richardson tableaux.

In Section 3 we formulate and prove the main result which is Theorem 3.1 , In Section 4 we present applications of Theorem 3.1. In particular, Corollary 4.3 states that each LR-tableau of a certain shape can be realized as the LR-tableau of a direct sum of cyclic embeddings. This property will allow us in [8] to study the partition of the variety of short exact sequences of nilpotent linear operators into its irreducible components.

\section{Direct sums of cyclic embeddings}

Let $\Lambda$ be a discrete valuation ring with maximal ideal generator $p$ and radical factor field $k$. By $\bmod \Lambda$ we denote the category of all (finite length) $\Lambda$ modules. It is well known that any finite length $\Lambda$-module $X$ is isomorphic to a direct sum

$$
X \cong \Lambda /\left(p^{\alpha_{1}}\right) \oplus \Lambda /\left(p^{\alpha_{2}}\right) \oplus \ldots \oplus \Lambda /\left(p^{\alpha_{n}}\right)
$$

where $\alpha_{1} \geq \alpha_{2} \geq \ldots \geq \alpha_{n}$. The assignment $X \mapsto \alpha=\left(\alpha_{1}, \ldots, \alpha_{n}\right)$ defines a bijection between the set of isomorphism classes of finite length $\Lambda$-modules and the set of all partitions. Given a partition $\alpha$, we denote by $N_{\alpha}(\Lambda)=N_{\alpha}$ the corresponding $\Lambda$-module.

Denote by $\mathcal{S}(\Lambda)$ the category of all short exact sequences in $\bmod \Lambda$ with morphisms given by commutative diagrams. With the componentwise exact structure, $\mathcal{S}(\Lambda)$ is an exact Krull-Remak-Schmidt category. We denote objects in $\mathcal{S}(\Lambda)$ either as short exact sequences $0 \rightarrow A \rightarrow B \rightarrow C \rightarrow 0$ of $\Lambda$-modules, or as embeddings $(A \subset B)$.

\subsection{Cyclic embeddings and poles}

We review Kaplansky's classification of cyclic embeddings.

An embedding $(A \subset B)$ is called cyclic if the submodule $A$ is cyclic as a $\Lambda$-module, that is, if $A_{\Lambda}$ is either indecomposable (i.e. of the form $\Lambda /\left(p^{n}\right)$ for some $n \geq 1)$ or zero. A cyclic embedding $(A \subset B)$ is a pole if $A$ is an 
indecomposable $\Lambda$-module and if $(A \subset B)$ is an indecomposable object in $\mathcal{S}(\Lambda)$. An embedding of the form $(0 \subset B)$ is called empty. By $E_{\beta}$ we denote the empty embedding $\left(0 \subset N_{\beta}\right)$.

For the classification we use height sequences which were originally introduced by Prüfer [12] as Höhenexponenten. In [4, Section 18], the height sequence for an element $a \in B$ is called its Ulm-sequence.

Definition: - A height sequence is a sequence in $\mathbb{N}_{0} \cup\{\infty\}$ which is strictly increasing until it reaches $\infty$ after finitely many steps. A height sequence is non-empty, if it has at least one element from $\mathbb{N}_{0}$.

- Let $B \in \bmod \Lambda$. We say an element $a \in B$ has height $m$ if $a \in p^{m} B$ $p^{m+1} B$. In this case we write $h(a)=m$. By definition, $h(0)=\infty$.

- The height sequence for $a$ in $B$ is $H_{B}(a)=\left(h\left(p^{i} a\right)\right)_{i \in \mathbb{N}_{0}}$. Usually, we do not write the entries $\infty$.

- A sequence $\left(m_{i}\right)$ has a gap after $m_{\ell}$ if $m_{\ell+1}>m_{\ell}+1$.

The following result is proved in [4, Theorem 25].

Proposition 2.1. There is a bijection

$$
\left.\{\text { poles }\} / \cong \stackrel{1-1}{\longleftrightarrow} \text { \{finite non-empty strictly increasing sequences in } \mathbb{N}_{0}\right\} \text {. }
$$

Example: Following [4, we construct a cyclic embedding $(A \subset B)$ for a given strictly increasing sequence $\left(m_{i}\right)_{0 \leq i \leq n}$ in $\mathbb{N}_{0}$. Let $i_{1}>i_{2}>\cdots>i_{s}$ be such that $\left(m_{i}\right)$ has gaps exactly after the entries $m_{i_{1}}>m_{i_{2}}>\cdots>m_{i_{s}}$. For $1 \leq j \leq s$ put $\beta_{j}=m_{i_{j}}+1$ and $\ell_{j}=m_{i_{j}}-i_{j}$, then $\beta$ and $\ell$ are strictly decreasing sequences of positive and nonnegative integers, respectively. Let

$$
B=N_{\beta}=\bigoplus_{i=1}^{s} \Lambda /\left(p^{\beta_{i}}\right)
$$

be generated by elements $b_{\beta_{j}}$ of order $p^{\beta_{j}}$. Let $a=\sum_{j=1}^{s} p^{\ell_{j}} \cdot b_{\beta_{j}}$ and put $A=(a)$. This yields a cyclic embedding $P\left(\left(m_{i}\right)\right)=(A \subset B)$.

Example: The height sequence $(1,3,4)$ has gaps after 1 and 4 , and hence gives rise to the embedding $P((1,3,4))=\left(\left(p^{2} b_{5}+p b_{2}\right) \subset N_{(5,2)}\right)$.

$$
P((1,3,4)): \stackrel{\square}{\square}
$$


In the picture, the columns correspond to the indecomposable direct summands of $B$; the $i$-th box from the top in a column of length $r$ represents the element $p^{i-1} b_{r}$. The columns are aligned vertically to make the submodule generator(s) homogeneous, if possible.

Lemma 2.2 (see [4, Lemma 22]). Suppose the height sequence $\left(m_{i}\right)$ of some element a in $B$ has a gap after $m_{\ell}$. Then $N_{\left(m_{\ell}+1\right)}$ occurs as a direct summand of $B$.

Proof of the lemma. See [4, Lemma 22 and page 29].

The following result is a slight generalization of Proposition 2.1. We will use it later.

Proposition 2.3. Every cyclic embedding in $\mathcal{S}(\Lambda)$ has the form

$$
P\left(\left(m_{i}\right)\right) \oplus E_{\beta}
$$

for a finite, possibly empty, strictly increasing sequence $\left(m_{i}\right)$ and a possibly empty partition $\beta$. More precisely, there is a one-to-one correspondence between the set of cyclic embeddings up to isomorphy and the set of pairs consisting of a finite, possibly empty, strictly increasing sequence and a partition.

Proof. Let $(A \subset B)$ be a cyclic embedding in $\mathcal{S}(\Lambda)$. The category $\mathcal{S}(\Lambda)$ has the Krull-Remak-Schmidt property so $(A \subset B)$ is isomorphic to a direct sum of indecomposable cyclic embeddings. As $\Lambda$ is a local ring, and as the submodule $A$ is indecomposable or zero, at most one of the summands has a nonzero submodule, hence is a pole, and hence is given uniquely, up to isomorphy, by a finite non-empty strictly increasing sequence in $\mathbb{N}_{0}$ (Proposition 2.1). (The case where the submodule $A$ is zero is included since the embedding given by the empty height sequence denotes the zero object in $\mathcal{S}(\Lambda)$.) The sum of the remaining indecomposable summands has the form $\left(0 \subset B^{\prime}\right)$ where $B^{\prime}$ is a $\Lambda$-module such that $\left(0 \subset B^{\prime}\right) \cong E_{\beta}$ for some partition $\beta$.

Remark: Each indecomposable cyclic embedding is either a pole of the form $P\left(\left(m_{i}\right)\right)$ for $\left(m_{i}\right)$ a finite non-empty strictly increasing sequence in $\mathbb{N}_{0}$ or else an empty embedding of the form $E_{(n)}$ for $n$ a natural number. 


\subsection{Partitions, tableaux and partial maps}

We introduce partial maps on Littlewood-Richardson tableaux and review cyclic embeddings.

Definition: Let $(\alpha, \beta, \gamma)$ be a partition triple. An LR-tableau of shape $\beta \backslash \gamma$ and content $\alpha$ is a Young diagram of shape $\beta$ (the outer shape of the tableau) in which the region $\beta \backslash \gamma$ is filled with $\left(\alpha^{\prime}\right)_{1}$ entries $1,\left(\alpha^{\prime}\right)_{2}$ entries 2, etc., where $\alpha^{\prime}$ denotes the transpose of $\alpha$, such that three conditions are satisfied:

- in each row, the entries are weakly increasing;

- in each column, the entries are strictly increasing;

- the lattice permutation property holds, that is, on the right hand side of each column there occur at least as many entries $e$ as there are entries $e+1(e=1,2, \ldots)$.

An embedding $(A \subset B)$ with corresponding short exact sequence $0 \rightarrow A \rightarrow$ $B \rightarrow C \rightarrow 0$ yields three partitions $\alpha, \beta, \gamma$ which describe the isomorphism types of $A, B, C$, respectively. The Green-Klein Theorem [5] states that a partition triple $(\alpha, \beta, \gamma)$ can be realized in this way by a short exact sequence if and only if there exists an LR-tableau of shape $\beta \backslash \gamma$ and content $\alpha$. The tableau of an embedding can be obtained in the following way.

Suppose that the submodule $A$ has Loewy length $r$. Then the sequence of epimorphisms

$$
B=B / p^{r} A \rightarrow B / p^{r-1} A \rightarrow \cdots \rightarrow B / p A \rightarrow B / A
$$

induces a sequence of inclusions of partitions

$$
\beta=\gamma^{(r)} \supset \gamma^{(r-1)} \supset \cdots \supset \gamma^{(1)} \supset \gamma^{(0)}=\gamma,
$$

where $N_{\gamma^{(e)}} \simeq B / p^{e} A$. The partitions define the corresponding LR-tableau $\Gamma=\left[\gamma^{(0)}, \gamma^{(1)}, \ldots, \gamma^{(r)}\right]$ as follows. The Young diagram of shape $\beta=\gamma^{(r)}$ is filled in such a way that the region given by $\gamma=\gamma^{(0)}$ remains empty and every box in the skew diagram $\gamma^{(e)} \backslash \gamma^{(e-1)}$ carries the entry $\boxminus$. The shape of the tableau is the skew diagram $\beta \backslash \gamma$, the content is the partition $\alpha$.

The union of two tableaux is taken row-wise, so if $\mathrm{E}=\left[\varepsilon^{(i)}\right]_{0 \leq i \leq s}$ and $\mathrm{Z}=$ $\left[\zeta^{(i)}\right]_{0 \leq i \leq t}$ are tableaux then the partitions $\gamma^{(i)}$ for $\Gamma=\mathrm{E} \cup \mathrm{Z}$ are given by taking the union (of ordered multi-sets): $\gamma^{(i)}=\varepsilon^{(i)} \cup \zeta^{(i)}$ where $\varepsilon^{(i)}=\varepsilon^{(s)}$ for $i \geq s$ and $\zeta^{(i)}=\zeta^{(t)}$ for $i \geq t$. 
Remark: It is easy to observe that if embeddings $(A \subset B),(C \subset D)$ have tableaux $\Gamma_{1}, \Gamma_{2}$, respectively, then the direct sum $(A \subset B) \oplus(C \subset D)$ has tableau $\Gamma=\Gamma_{1} \cup \Gamma_{2}$.

Definition: Column tableaux (which we call columns if the context is clear) are tableaux which have only one column, and where the entries in this column are subsequent natural numbers (not necessarily starting at 1). Each column is one of the following: For $1 \leq e \leq f$, we denote by $C(e, f)_{n}$ the 1-column tableau of height $n$ with entries $e, \ldots, f$. Formally, $C(e, f)_{n}=$ $\left[\gamma^{(0)}, \ldots, \gamma^{(f)}\right]$ where $\gamma^{(0)}=\cdots=\gamma^{(e-1)}=(n-f+e-1), \gamma^{(e)}=(n-f+$ $e), \ldots, \gamma^{(f)}=(n)$. By $C(1,0)_{n}$ we denote the empty column of length $n$. Note that a column tableau $C(e, f)_{n}$ satisfies the lattice permutation property, and hence is an LR-tableau, if and only if $e=1$.

Example: In a union of column tableaux, the vertical columns need no longer form column tableaux.

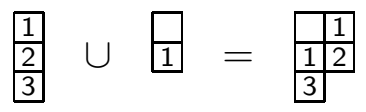

Definition: A partial map $g$ on an LR-tableau $\Gamma$ assigns to each box @ with $e>1$ a box d with entry $d=e-1$ such that

1. $g$ is one-to-one,

2. for each box $b$, the row of $g(b)$ is above the row of $b$.

An orbit of a partial map $g$ is a sequence $\boxminus, g(\boxminus), g^{2}(\boxminus), \ldots, g^{e-1}(\boxminus)$ given by a box $€$ which is not in the image of $g$. Thus, the orbits of $g$ are in one-to-one correspondence with the boxes $\square$ not in the image of $g$ (the correspondence is given by taking the first box in the orbit), and in one-to-one correspondence with the boxes that have entry 1 (where the correspondence is given by taking the last box).

Remark: Given an LR-tableau $\Gamma$, the existence of at least one partial map follows from the lattice permutation property.

Given a partial map $g$ on an LR-tableau $\Gamma$, a jump in row $r$ is a box $b$ in this row with the property that either $b$ has entry 1 or that $g(b)$ occurs in row $s$ with $s<r-1$. We say a partial map $g$ on $\Gamma$ has the empty box property $(E B P)$ if for each row $r$, there are at least as many columns in $\Gamma$ of exactly $r-1$ empty boxes as there are jumps in row $r$. 
Example: Consider the following LR-tableau

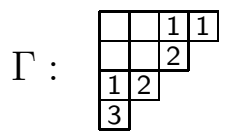

To define a partial map, we have to assign to each box 3 a corresponding box 2 and to each box 2 a corresponding box 1 . Note that we can do this in four different ways. To specify the maps, we distinguish boxes of the same entry by indicating the row on the right; for the two boxes in the first row, we use the letters $\mathrm{L}$ and R. Consider the partial map $g$ defined as follows:

$$
g: 3^{-4} \mapsto 2^{-3}, 2^{-3} \mapsto\left[1-\left\llcorner, \quad 2-2 \mapsto{ }^{-R}\right.\right.
$$

Note that the partial map $g$ has three orbits (one consists only of the box $1 \mathbf{1}^{3}$ ). It satisfies (EBP) since there is a column of 2 empty boxes corresponding to the jump $2^{-3} \mapsto 1^{-1}$. However, the partial map

$$
g^{\prime}: \text { 3-4 }
$$

does not satisfy (EBP), because there is no column of 3 empty boxes corresponding to the jump at $35^{-4} \mapsto 22^{-2}$.

We collect some properties of tableaux for cyclic embeddings.

Proposition 2.4. Suppose the embedding $(A \subset B)$ is cyclic with $A$ a module of Loewy length $r$.

1. In the tableau, each entry $1, \ldots, r$ occurs exactly once.

2. The height sequence $\left(m_{i}\right)$ of the submodule generator a determines the rows in $\Gamma$ in which the entries occur, and conversely. More precisely, the entry e occurs in row $m_{e-1}+1$.

3. The LR-tableau $\Gamma$ of a cyclic embedding is a union of columns with disjoint entries.

4. There is a unique partial map on $\Gamma$; it satisfies the (EBP).

Proof. 1. For the first statement note that for each $0<e \leq r$, the module $B / p^{e-1} A$ is a factor module of $B / p^{e} A$ of colength one. 
2. For any embedding, the number of boxes in the first $m$ rows of $\gamma^{(e)}$ is the length of $\left(B / p^{e} A\right) /\left(\left(p^{m} B+p^{e} A\right) / p^{e} A\right)=B /\left(p^{e} A+p^{m} B\right)$. Here, the modules $B /\left(p^{e-1} A+p^{m} B\right)$ and $B /\left(p^{e} A+p^{m} B\right)$ have the same length if $m \leq m_{e-1}$ and different lengths otherwise since $p^{e-1} a \in p^{m_{e-1}} B \backslash p^{m_{e-1}+1} B$.

3. By construction of $P\left(\left(m_{i}\right)\right)$, each gap in the height sequence gives rise to a new summand of $B$ and hence to a new column in the tableau. It follows from 2. that $\Gamma$ is a union of columns.

4. There is a unique partial map on $\Gamma$ given by assigning to a box $\boxminus$ where $e>1$ the unique box with entry $e-1$. Since $\Gamma$ is a union of columns, the (EBP) is satisfied.

COROLlary 2.5. 1. There is a one-to-one correspondence between cyclic embeddings up to isomorphy and LR-tableaux in which each entry occurs exactly once.

2. Under this correspondence, a pole corresponds to a tableau in which the number of columns equals the number of gaps in the height sequence. An empty embedding $E_{\beta}$ corresponds to the empty tableaux on the Young diagram for $\beta$.

Proof. 1. By the Green-Klein theorem, any LR-tableau $\Gamma$ can be realized as the tableau of an embedding $(A \subset B)$. If $\Gamma$ has exactly one box 1 , then $A$ is an indecomposable $\Lambda$-module since $\operatorname{dim} A / p A=\operatorname{dim} B / p A-\operatorname{dim} B / A=$ $\#\{$ boxes 1$\}$. The height sequence of the generator of $A$ can be read off using Proposition 2.4. 2. The uniqueness of the isomorphism type follows from Proposition 2.3.

2. Regarding poles, we have seen in the proof of Proposition 2.1 that the summands of the ambient space of a pole correspond to the gaps in the height sequence of the submodule generator. The result follows from Proposition 2.3.

\section{Direct sums of cyclic embeddings}

We can now generalize Kaplansky's results and give a combinatorial description for finite direct sums of cyclic embeddings, up to isomorphy.

Definition: - Two partial maps $g, g^{\prime}$ on an LR-tableau $\Gamma$ are equivalent if $g^{\prime}=\pi^{-1} \circ g \circ \pi$ for some permutation $\pi$ on the set of non-empty boxes in $\Gamma$ which preserves the entry and the row. 
- Two pairs $(\Gamma, g),(\Delta, h)$, each consisting of an LR-tableau and a partial map on the tableau, are equivalent if $\Gamma=\Delta$ and if the partial maps $g, h$ are equivalent. In this case we write $(\Gamma, g) \sim(\Delta, h)$.

Example: Consider the LR-tableau from the example in Section 2.2.

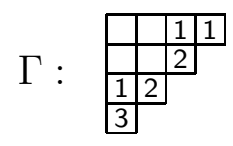

The maps $g$ and $g^{\prime}$ are not equivalent. But $g$ is equivalent to the partial map

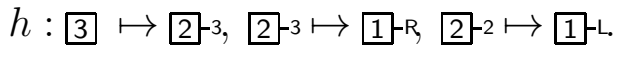

(There is a fourth partial map on $\Gamma$, it is equivalent to $g^{\prime}$.)

Clearly, given two equivalent partial maps on an LR-tableau, then one satisfies (EBP) if and only if the other does.

THEOREM 3.1. There is a one-to-one correspondence

$$
\{\bigoplus \text { cyclics }\} / \cong \stackrel{1-1}{\longleftrightarrow}\{(\Gamma, g): \text { g partial map on } \Gamma \text { with }(E B P)\} / \sim
$$

between the isomorphism types of direct sums of cyclic embeddings, and the equivalence classes of pairs $(\Gamma, g)$ where $\Gamma$ is an LR-tableau and $g$ a partial map on $\Gamma$ which satisfies (EBP).

Proof. We describe this correspondence explicitely.

Consider a direct sum of cyclic embeddings as the sum of an empty embedding and a direct sum of poles (Proposition 2.3). The height sequence of each pole gives rise to a partial map on the tableau of the pole which satisfies (EBP), this map has precisely one orbit; it records the isomorphism type of the pole (Proposition 2.4). The tableau of the direct sum is given by taking the row-wise union of the tableaux of the summands; it admits a partial map which is (boxwise) defined by the partial maps for the summands; this map satisfies (EBP) because the restricted maps do. Given two isomorphic direct sum decompositions, the two unordered lists of height sequences of the poles involved are equal, hence the two associated partial maps differ by conjugation by a permutation of the boxes which preserves rows and entries. Conversely, suppose $g$ is a partial map on $\Gamma$ with (EBP). For each orbit $\mathcal{O}$ of $g$, the boxes in $\mathcal{O}$ together with the empty parts of columns which correspond 
to the jumps (recall the definition of the (EBP)), constitute the tableau of a pole. The empty parts of columns in $\Gamma$ not used by the (EBP) define the tableau of an empty embedding. Since all boxes are accounted for, $\Gamma$ is the union of all the tableaux. Hence the sum of the poles and the empty embedding has $\Gamma$ as its tableau. If $g^{\prime}$ is an equivalent partial map on $\Gamma$, then the boxes in the orbits may differ, but not the rows in which they occur. Thus the corresponding unordered list of height sequences of poles is equal, and so is the partition which records those empty parts of columns which are not used up by the jumps in the height sequences. Hence the associated embeddings are isomorphic.

The two assignments are inverse to each other since this is true for poles and empty embeddings.

There is a different way to read off from a given LR-tableau if it is the tableau of a direct sum of cyclic embeddings.

Corollary 3.2. An LR-tableau $\Gamma$ is the tableau of a direct sum of cyclic embeddings if and only if $\Gamma$ is a union of columns.

Proof. In Proposition 2.4 we have seen that the tableau of a cyclic embedding is a union of columns, and this property is preserved under taking direct sums. Conversely, if the tableau is a union of columns, then the lattice permutation property allows for the construction of a partial map with (EBP). The result follows from Theorem 3.1 .

We conclude this section with examples and remarks.

Example: In general, the embedding corresponding to a given LR-tableau is not determined uniquely, up to isomorphy, by the tableau alone. In each case, the following embeddings will have the same given LR-tableau.

(1) Two nonisomorphic sums of poles, given by nonequivalent partial maps.

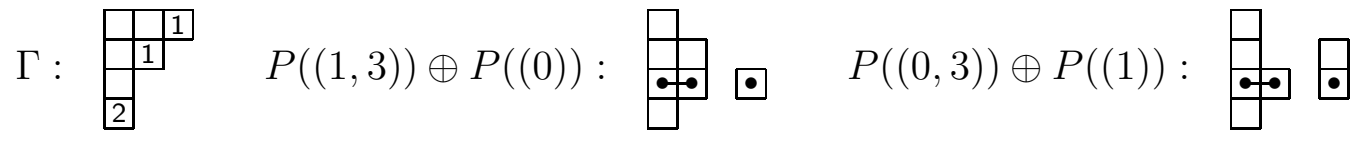

(2) A sum of two poles vs. a sum of two poles and an empty embedding.

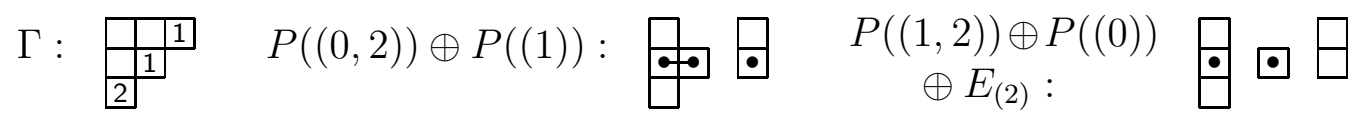


(3) The sum of poles is determined uniquely by the partial map; but there is also an embedding which is not a sum of poles.

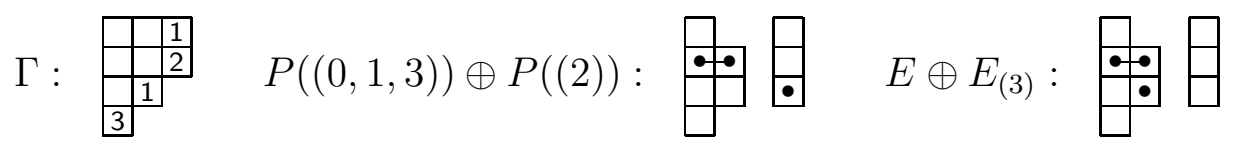

(4) Two partial maps, up to equivalence, only one has the (EBP).

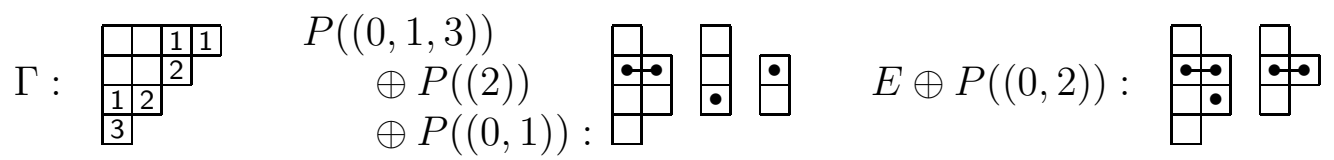

(5) Two partial maps with (EBP), up to equivalence.

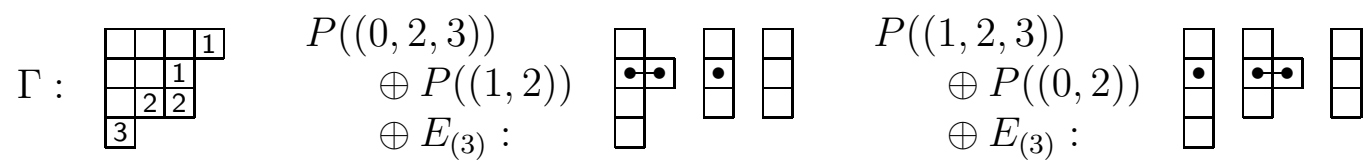

Remark: The proof of Theorem 3.1 shows how to read off the direct sum decomposition from a given pair $(\Gamma, g)$. Each orbit of $g$ gives rise to a pole, its height sequence is given by Proposition 2.4. To each jump in the height sequence, there is a corresponding empty part of a column in $\Gamma$. The empty boxes in those columns which do not correspond to any pole determine the remaining empty embedding.

Remark: An algebraic description of finite direct sums of cyclic embeddings is given in [3, in particular Lemma 1 and Theorem 2]. Here, the multiplicity of a pole $P$ as a direct summand of a given embedding $M$ is the dimension of the $k$-vector space $F_{P}(M)$, for a suitable functor $F_{P}: \mathcal{S}(\Lambda) \rightarrow \bmod k$.

\section{Applications of height sequences}

We briefly describe four situations in which height sequences or direct sums of cyclic embeddings play a role.

\section{1 $\quad p^{2}$-bounded embeddings in infinite length modules}

In this paragraph, $B$ may be any $\Lambda$-module, not necessarily finitely generated. 
Corollary 4.1. Suppose that $(A \subset B)$ is an embedding of $\Lambda$-modules where $A$ is $p^{2}$-bounded and where $B$ is either finitely generated or bounded. Then the embedding is a direct sum of cyclic embeddings.

Proof. The case where $B$ is finitely generated is covered in [11, Corollary 5.4]. There is exactly one indecomposable embedding where $B$ is not bounded, namely $(0 \subset \Lambda)$.

For the bounded case, note that an embedding $(A \subset B)$ with $p^{2} A=0=p^{n} B$ can be considered a module over the ring

$$
R=\left(\begin{array}{cc}
\Lambda /\left(p^{2}\right) & \Lambda /\left(p^{2}\right) \\
0 & \Lambda /\left(p^{n}\right)
\end{array}\right),
$$

so [10, Theorem 1] yields the claim.

\subsection{Direct sums of cyclics in the Green-Klein Theorem}

Let $\alpha, \beta, \gamma$ be partitions. Recall from [5] that there exists a short exact sequence

$$
\mathcal{E}: 0 \rightarrow N_{\alpha} \rightarrow N_{\beta} \rightarrow N_{\gamma} \rightarrow 0
$$

if and only if there exists an LR-tableau of shape $\beta \backslash \gamma$ and content $\alpha$. As a consequence, we obtain

Corollary 4.2. Given partitions $\alpha, \beta, \gamma$, there exists an embedding $N_{\alpha} \rightarrow$ $N_{\beta}$ with cokernel $N_{\gamma}$ which is a direct sum of cyclic embeddings if and only if there is an LR-tableau of shape $\beta \backslash \gamma$ and content $\alpha$ which is a union of columns.

Proof. Using Corollary 3.2 it is possible to decide whether there exists an embedding $N_{\alpha} \rightarrow N_{\beta}$ with cokernel $N_{\gamma}$ which is a direct sum of cyclic embeddings.

\subsection{Tableaux which are horizontal strips}

The skew diagram $\beta \backslash \gamma$ is said to be a horizontal strip if $\beta_{i} \leq \gamma_{i}+1$ holds for all $i$, and a vertical strip if $\beta^{\prime} \backslash \gamma^{\prime}$ is a horizontal strip. We are particularly interested in the following situation. 
COROLlary 4.3. Suppose $\Gamma$ is an LR-tableau of shape $(\alpha, \beta, \gamma)$ such that $\beta \backslash \gamma$ is a horizontal strip. Then there exists an embedding $(A \subset B)$ which is a direct sum of cyclic embeddings and which has tableau $\Gamma$.

Proof. Since $\Gamma$ is an LR-tableau, there exists a partial map $g$ on $\Gamma$. Since $\beta \backslash \gamma$ is a horizontal strip, each column in the tableau $\Gamma$ has at most one entry, so the (EBP) is trivially satisfied. The embedding corresponding to the partial map $g$ is a direct sum of cyclic embeddings, and it has the tableau $\Gamma$.

Remark: In the situation of Corollary 4.3, finite direct sums of cyclic embeddings turn out to be a useful tool for the study of the variety $\mathbb{V}_{\alpha, \gamma}^{\beta}$ of all embeddings $N_{\alpha} \rightarrow N_{\beta}$ with cokernel $N_{\gamma}$; here, $\Lambda=k[[T]]$ is the power series ring with coefficients in an algebraically closed field. Namely, each irreducible component of $\mathbb{V}_{\alpha, \gamma}^{\beta}$ has the form $\overline{\mathbb{V}}_{\Gamma}$ for some LR-tableau $\Gamma$ of shape $(\alpha, \beta, \gamma)$. The boundary relation for tableaux $\Gamma, \Delta$ is given by the condition that $\overline{\mathbb{V}}_{\Gamma} \cap \mathbb{V}_{\Delta} \neq \emptyset$. In [8, Theorem 5.1] it is shown that box moves in LR-tableaux imply the boundary relation by constructing one-parameter families of embeddings which are all finite direct sums of cyclic embeddings.

\subsection{Endo-submodules of $\Lambda$-modules}

Given a $\Lambda$-module $B$, we obtain a precise description for the submodules of $B$ when considered as a module over its endomorphism ring.

We recall from [4, Theorem 24] that a height sequence $\left(m_{i}\right)$ defines an $\operatorname{End}(B)$ submodule consisting of all elements of $B$ with height sequence $\geq\left(m_{i}\right)$ (this is the "fully transitive" property). The partial ordering on height sequences is given by $\left(m_{i}\right) \geq\left(q_{i}\right)$ if $m_{i} \geq q_{i}$ holds for all $i \in \mathbb{N}$.

It is shown in [4, Theorem 25] that every $\operatorname{End}(B)$-submodule of $B$ is cyclic (as an $\operatorname{End}_{\Lambda}(B)$-module), and hence is determined uniquely, up to equality, by the height sequence of a generator.

Suppose $a \in B$ has height sequence $\left(m_{i}\right)$ with gaps exactly after $m_{i_{1}}>$ $m_{i_{2}}>\cdots>m_{i_{s}}$. We can specify the corresponding $\operatorname{End}(B)$-submodule $X$ explicitly. Namely, with $a=\sum_{j} p^{\ell_{j}} b_{\beta_{j}}$ as above, also the summands $p^{\ell_{j}} b_{\beta_{j}}$ are in $X$, and so are their images under endomorphisms of $B$. Put $k_{j}=\beta_{j}-\ell_{j}$ 
for $1 \leq j \leq s$, then

$$
\operatorname{End}(B) \cdot a=\sum_{j=1}^{s}\left(\operatorname{rad}^{\ell_{j}} B \cap \operatorname{soc}^{k_{j}} B\right)
$$

With this notation, the endo-submodule $\operatorname{End}(B) \cdot a$ can also be written as an intersection of sums:

$\operatorname{End}(B) \cdot a=\operatorname{rad}^{\ell_{1}} B \cap\left(\operatorname{soc}^{k_{1}} B+\operatorname{rad}^{\ell_{2}} B\right) \cap \cdots \cap\left(\operatorname{soc}^{k_{s-1}} B+\operatorname{rad}^{\ell_{s}} B\right) \cap \operatorname{soc}^{k_{s}} B$

To verify this, use induction and the modular law.

The following result leads to a quick proof for the formula for the number of $\operatorname{End}(B)$-submodules of a finite length $\Lambda$-module $B$.

Corollary 4.4. Suppose the $\Lambda$-module $B$ is given by a partition $\beta$. Any two of the following sets are in one-to-one correspondence.

1. The set of $\operatorname{End}(B)$-submodules of $B$.

2. The set of embeddings of the form $((a) \subset B)$, up to isomorphy.

3. The set of LR-tableaux with outer shape $\beta$ in which each entry occurs exactly once.

As a consequence, the $\Lambda$-module $B=N_{\beta}$ has exactly $\prod_{i}\left(1+\beta_{i}-\beta_{i+1}\right)$ many endo-submodules.

Proof. In each of the sets, the elements are given by height sequences. The correspondence follows from Corollary 2.5, and Proposition 2.4, 2., together with the isomorphy between the lattices of $\operatorname{End}(B)$-submodules of $B$ and of height sequences of elements in $B$ in [4, Theorem 25]. For the formula we count the third set. Note that tableau properties require that each entry be the rightmost entry in its row since there are no multiple entries. Hence in the $i$-th column, there may be between 0 and $\beta_{i}-\beta_{i+1}$ many entries. The expression is the product of the number of choices for the number of entries in each column.

Dedication. The authors wish to dedicate this paper to Professor José Antonio de la Peña on the occasion of his 65 th birthday. Throughout their 
careers, Professor de la Peña has shown an active interest in their work, supported them through invitations to important conferences, and inspired them through his dedication to representation theory and his interest in applications. His exemplary paper [2] has been a model for this manuscript since combinatorial invariants control families of modules which turn out to play a meaningful role geometrically.

\section{References}

[1] G. Birkhoff, Subgroups of abelian groups, Proc. Lond. Math. Soc., II. Ser. 38 (1934), 385-401.

[2] T. Brüstle, J. A. de la Peña and A. Skowroński, Tame algebras and Tits quadratic forms, Advances in Mathematics 226 (2011), 887-951.

[3] R. Hunter, F. Richman and E. A. Walker, Finite direct sums of cyclic valuated p-groups, Pacific J. Math. 69, 97-104 (1977).

[4] I. Kaplansky, Infinite abelian groups, University of Michigan Press, 1954.

[5] T. Klein, The multiplication of Schur-functions and extensions of $p$ modules, J. Lond. Math. Soc. 43 (1968), 280-284.

[6] J. Kosakowska and M. Schmidmeier, Operations on arc diagrams and degenerations for invariant subspaces of linear operators, Trans. Amer. Math. Soc. 367, 5475-5505 (2015).

[7] J. Kosakowska and M. Schmidmeier, Arc diagram varieties, in: Expository Lectures on Representation Theory, Contemp. Math. 607, 205223 (2014).

[8] J. Kosakowska and M. Schmidmeier, Box moves on Littlewood-Richardson tableaux and an application to invariant subspace varieties, J. Alg. 491 (2017), 241-264.

[9] J. Kosakowska and M. Schmidmeier, The boundary of the irreducible components for invariant subspace varieties, Math. Zeit. 290 (2018), 953-972. 
[10] A. Moore, The Auslander and Ringel-Tachikawa Theorem for submodule embeddings, Comm. Alg. 38, 3805-3820 (2010).

[11] C. Petroro and M. Schmidmeier, Abelian groups with a $p^{2}$-bounded subgroup, revisited, J. Alg. Appl. 10, 377-389 (2011).

[12] H. Prüfer, Abzählbare primäre Abelsche Gruppen, Math. Zeit. 17, 3561 (1923).

Address of the authors:

Faculty of Mathematics and Computer Science

Nicolaus Copernicus University

ul. Chopina 12/18

87-100 Toruń, Poland

justus@mat.umk.pl
Department of Mathematical Sciences Florida Atlantic University 777 Glades Road

Boca Raton, Florida 33431

markus@math.fau.edu 